\title{
A Novel Device to Insert FU Grafts into Premade Sites
}

\author{
Parsa Mohebi, MD, FISHRS I Los Angeles, California, USA I info@parsamohebi.com
}

Disclosure: The Mohebi Inserter was invented by Dr. Parsa Mohebi. Dr. Mohebi receives royalty from the manufacturer.

\section{INTRODUCTION}

Hair transplantation surgery has dramatically improved over the past decade thanks to the emergence of new techniques of follicular unit transplantation (FUT) and follicular unit excision (FUE) allowing for greater efficiency and improved safety.

Sharp implanter devices have been developed because in some countries both the recipient site incision and also the graft insertion has to be performed by the surgeon personally. The popularity of FUE hair transplantation has provoked the development of more advanced devices that potentially increase the speed of the hair transplant procedure. We are now able to perform larger FUE procedures in shorter timespans. Multiple options regarding punch devices have evolved in addition to progress in implanter technology.

The reduction in the size of the punch and the extraction part of the procedure both result in more fragile grafts versus those grafts obtained via strip harvesting. As a result, careful handling of the FUE grafts with forceps may be difficult or too slow. Many surgeons think that implanters may minimize injury to these delicate grafts while also increasing the speed of the procedure.

\section{Problem}

Over the past few years, great strides have been made to expedite and enhance the FUE process. As the number of patients seeking FUE continues to grow, many hair transplant surgeons recognize the need for this technique while also embracing its current shortcomings and developing more advanced technologies.

Standard handling includes forceps to hold and insert robust strip FUT grafts. However, handling fragile, skinny FUE grafts and placing them using forceps, in my opinion, could jeopardize the growth of the grafts by applying unnecessary stress upon them. The solution was to develop a method of excision and placing that would minimize a graft's time out of the body. With the advent of simultaneous graft handling, grafts could be excised, counted, sorted, and placed via a rapid continuous circular flow. This method necessitated that sites be made by the surgeon prior to graft excision. The serial excision and placement loop began with the surgeon creating recipient sites and then harvesting grafts, so the placing team could insert them into the premade sites using a new type of dull, non-incisional implanters shortly thereafter. This method potentially minimized the time the grafts remained out of body.

Sharp implanters were initially widely used but they required a team of one or two technicians to manually load the grafts into the implanters before the surgeon could simultaneously incise the skin and implant the grafts. This resulted in the procedure becoming even more dependent upon the surgeon as use of the sharp tip required the surgeon to incise the skin and insert grafts personally. Despite potentially reducing the overall time of the procedure, this device increased the time the surgeon had to spend in the operating room. To reduce the common problem of buried grafts, Dr. Tommy Hwang invented the sharp Hwang Implanter, which also had a depth control that facilitated the controlled insertion of grafts by the surgeon.

Next came dull implanters, introduced by Mauro Speranzini. Dull implanters possessed a distinct advantage over sharp implanters because they did not require the surgeon to insert the grafts. In most countries, graft placing into premade sites can be done by technical assistants. Instead, incisions were carefully premade in advance by the surgeon allowing one or two technicians to load the grafts while others inserted them. This setup still required two groups of technicians with one group as loaders and another group as placers.

In an effort to reduce the number of technicians and steps involved in placing the grafts, Sajeev Vasa's SAVA ${ }^{\circledR}$ Implanter and Koray Erdogan's KEEP (Koray Erdogan Embedding Placer) offered a simple twist to the dull implanters by combining the benefits of a blunt tip with a single tech loading mechanism. This compact, easy-to-handle device allowed each technician to load and place their own grafts into premade sites created by the surgeon, just as they would do traditionally with forceps placing. Once in the site, each graft was guided along the length of a narrow groove with the aid of forceps.

The KEEP furthered the concept of minimizing the number of technicians encircling a patient's head. It allowed for simultaneous excision and placement of grafts into premade sites. One drawback to the KEEP compared to forceps placing alone or pre-loaded implanters, though, was that each technician still had to use both hands because the placing person needed to hold the device in one hand while guiding the graft with a forceps held in the other hand. Despite using the KEEP, the area around the patient's head was still a crowded field as it did not allow more than one placer to insert the grafts into premade sites while the surgeon and his or her assistants were simultaneously harvesting grafts.

To further improve this concept, we developed a graft inserter that could be loaded and deployed with only one hand by the same technician (Figures 1 and 2). We decided to call it "inserter" to further emphasize that it is a dull, nonincisional device and not a surgical instrument to make incisions such as a sharp implanter. Therefore, in most countries, it can be used by assistants.

The development and
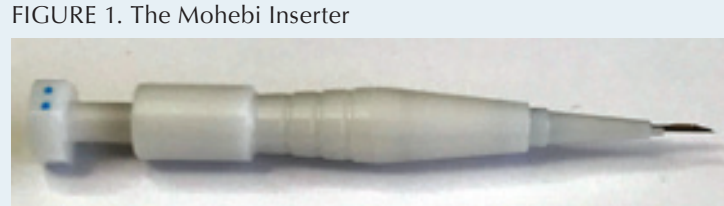

IGURE 2. Close-up of tip 
design advancement of the inserter took into account that the area around the patient's head is premium space. The compact Mohebi Inserter allows for reduced elbow room. Thus, the excising surgeon, the graft-extracting technician, and two placers equipped with the Mohebi Inserter are able to perform their task in limited space. This inserter allows technicians to do the following:

- Load their own grafts (Figures 3 and 4).

- Use only one hand for inserting the grafts into premade sites (Figures 5 and 6).

- Comfortably switch hands depending of the angle of the sites.

- Keep their distance from the patient's head allowing for simultaneous graft excision and placing into premade sites.

FIGURE 3. Technicians arrange the grafts on their gloves (left). Grafts are arranged all in one direction (right).

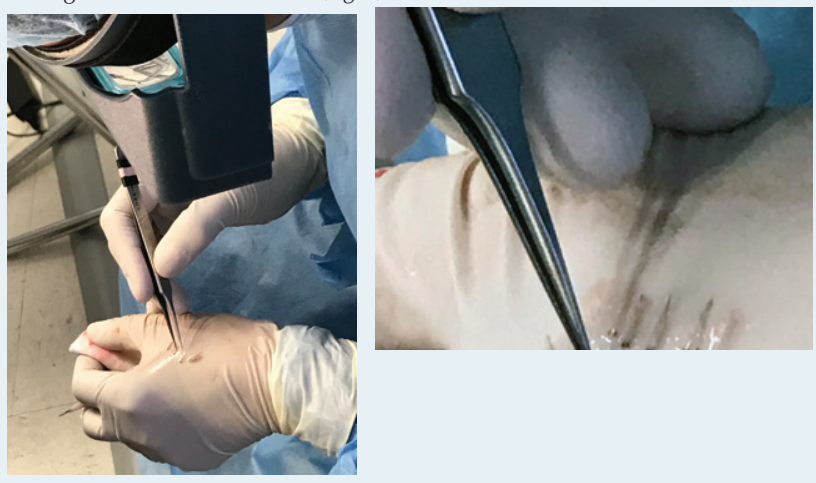

FIGURE 4. Inserter orientation (left). Inserter slot is placed over the bulb of the graft (center). Inserter is retracted so the graft gets inserted into the lumen (right).
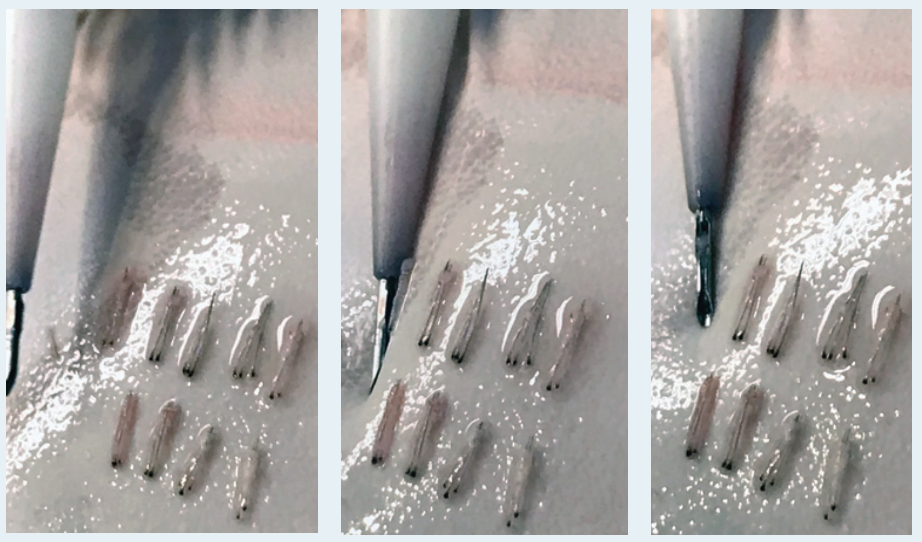

FIGURE 5. The tip of the inserter is inserted into the incision (left) and the inserter is advanced into the incision (right).
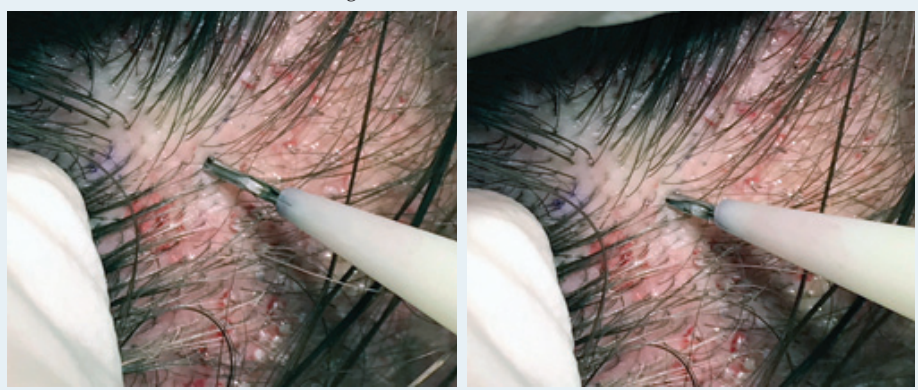

FIGURE 6. The inserter's plunger is pushed to implant the graft (left) and the graft is implanted and the inserter is removed (right).
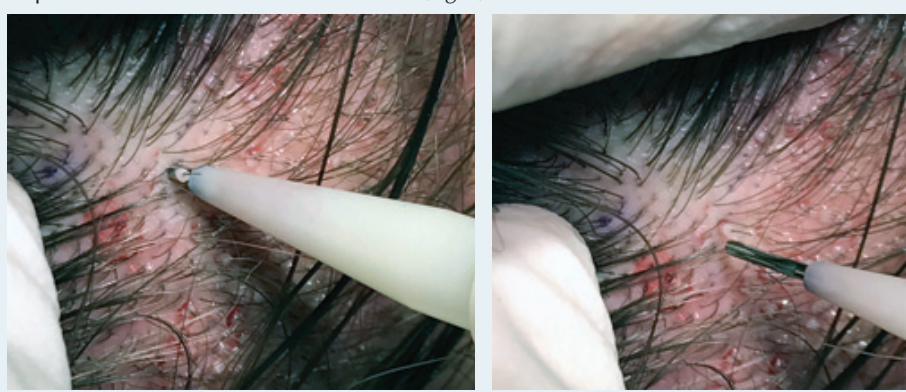

\section{Solution}

The Mohebi Inserter possesses a narrow slot through which technicians may load their own grafts before placing them just by rolling the inserter over the grafts. Previous devices either had to be pre-loaded or required the use of two hands. Simple modifications, such as using loupes with a long focal point, allow for an adequate distance from the patient's head during placement. Maintaining an arm's-length working distance and the use of a single-hand implanter have given way to allowing for simultaneous excision and placement into premade sites. With its one-person, one-hand design, the Mohebi Inserter offers increased space around the patient and makes inserting more efficient.

\section{HOW THE MOHEBI INSERTER WORKS Loading}

The Mohebi Inserter has a unique loading system that can accommodate grafts of varying sizes. During graft loading, the technician holds the device in the dominant hand while grafts are arranged in a tidy row over the other hand. A brief pre-sorting on the front end helps expedite loading the grafts into the inserter. The inserter shaft has a unique teardrop-shaped slot on one side that is rotated downward to hover over the bulbs of the follicles. The inserter is then lowered so the bulbar ends of the grafts enter the opening. The next step is to gently push down and pull the inserter back, so that the grafts can smoothly enter the lumen of the inserter. A correctly loaded inserter has the bulbar end of the graft lying well protected within the lumen of the inserter while the graft's epidermal side can be out and visible.

\section{Placing}

After loading a graft, the dull tip of the graft inserter is gently inserted into the incision previously made by the surgeon without deploying the plunger. The technician can insert the entire dull needle of the inserter into the incision in order to identify the track of the premade incision before deploying the plunger. The other option would be to insert only the distal $1 \mathrm{~mm}$ of the inserter tip before deploying the plunger.

When the inserter is positioned properly inside the incision, the plunger is deployed directing the graft swiftly inside the incision. The opening on top of the inserter is held upward during deployment of the plunger so the technician can visualize the location of the graft and be assured of the graft's proper deployment. Once the graft is in position, the technician needs to rotate the inserter such that the skin 
cap lies parallel to the scalp. Depth control (designed by Dr. Tommy Hwang) prevents the grafts from being buried. The graft skin cap must be oriented parallel to the skin and slightly above the surface. This allows the curvature of the grafts to follow a natural curve of the hair follicle in the area.

\section{Advantages}

The Mohebi Inserter has many benefits by virtue of its easy-to-master technical aspect; tight space, single-person design; and compact handle. This inserter is designed to deliver consistent and reliable results. One of the most important issues is minimizing the space that every technician needs around the patient's head. Given that the Mohebi Inserter can be loaded and deployed with only one hand, the predicament of limited space becomes inconsequential as the fight over elbow room and workable space is not an issue.

\section{SUMMARY}

The Mohebi Inserter is a dull implanter designed to potentially reduce the manipulation of the hair follicles during the placement process into premade sites. See our instruction video at: http://parsamohebi.com/resources/drmohebis-innovations/inserter. Having premade sites allows two technicians to start placing while the surgeon and a technician harvest the grafts. One-handed placement of the grafts with the Mohebi Inserter allows the technicians to further distance themselves from the patient while decreasing the space to perform placement around the patient's head. This much needed space is crucial in maximizing the number of placers that can fit around the patient's head, and this is particularly true during the simultaneous excision and placement process.
The Mohebi Inserter was developed in response to the need for a device that could load and deploy grafts into premade recipient sites by a single user. This inserter borrows its plunger from the traditional sharp implanter, exhibits similarities to the Vasa and KEEP graft placers, and uses a similar mechanism for depth control as the Hwang Implanter. The inserter has a short learning curve, which allows new technicians to quickly become efficient. The Mohebi Inserter may become a useful addition to the arsenal of tools needed to successfully perform an FUE procedure.

Editor's note: This is an interesting new device combining and improving ideas from other placing instruments. We are seeing more and more instruments for FUE harvesting and placing. However, many claims have not been proven. We rarely see long-term results. For harvesting instruments, we need transection rates and data about the graft quality. For placing devices, we need data regarding cosmetic outcome and survival rates. Does increased speed and simultaneous harvesting and placing impair the quality of the procedure? Is there really such a thing as a one-hand device? Are grafts injured or squeezed during loading and placing? Is the use of implanters actually an improvement over forceps placing? What are the survival rates and complications compared to forceps, which allow the placer to feel the recipient site and see the graft while placing?

Many users of forceps have developed great skill to place grafts. They like the fact that it allows them to rate and choose the graft and place it into the most appropriate area according to the cosmetic preferences. They also like that the grafts can be grasped and put in the desired direction without major manipulation or twisting. Is this possible with other placing instruments? - AF $\square$

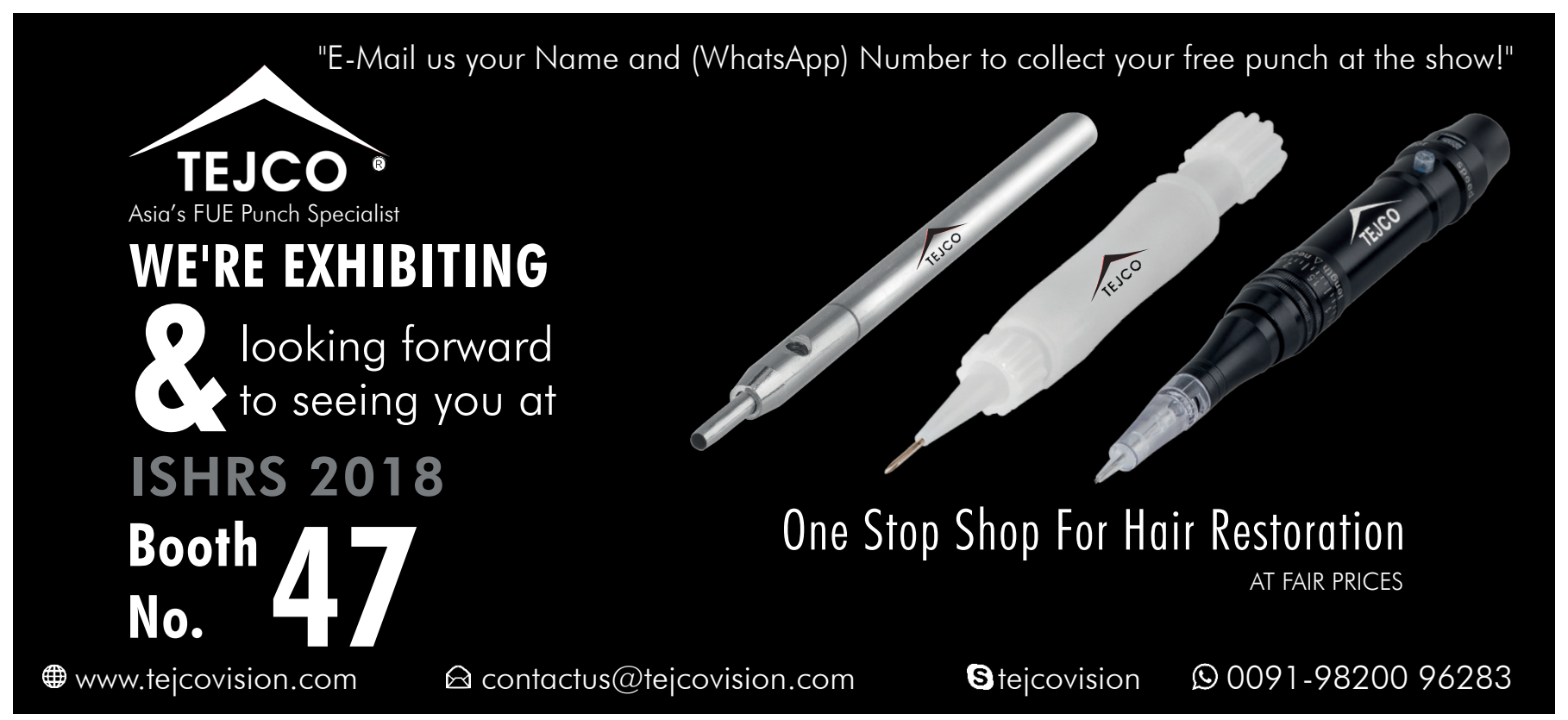

